Research Paper

\title{
Circular RNA Hsa_circ_0066755 as an Oncogene via sponging miR-651 and as a Promising Diagnostic Biomarker for Nasopharyngeal Carcinoma
}

\author{
Jian Wang ${ }^{\circledR}$, Jinyu Kong ${ }^{2}$, Zhong Nie ${ }^{1}$, Diansen Chen ${ }^{1}$, Jun Qiang1, Wanqin Gao ${ }^{1}$ and Xiaojie Chen ${ }^{3}$ \\ 1. Center of Image Diagnoses, The First Affiliated Hospital, and College of Clinical Medicine of Henan University of Science and Technology, Luoyang 471003, \\ China. \\ 2. Henan Key Laboratory of Cancer Epigenetics; Cancer Institute, The First Affiliated Hospital, and College of Clinical Medicine of Henan University of \\ Science and Technology, Luoyang 471003, China. \\ 3. Medical College, Henan University of Science and Technology, Luoyang 471003, Henan, China.
}

$\square$ Corresponding author: Dr. Jian Wang, The First Affiliated Hospital, and College of Clinical Medicine of Henan University of Science and Technology. No.24 Jinghua Road, Jianxi District, Luoyang 471003, China; Tel./Fax: +86 0379 69823173; E-mail: jwlab2000@126.com.

(c) The author(s). This is an open access article distributed under the terms of the Creative Commons Attribution License (https://creativecommons.org/licenses/by/4.0/). See http:/ /ivyspring.com/terms for full terms and conditions.

Received: 2020.04.14; Accepted: 2020.06.02; Published: 2020.06.15

\begin{abstract}
Background: Circular RNAs (circRNAs) represent a class of broad and diversified endogenous RNAs that regulate gene expressions in eukaryotes. Hsa_circ_006675 has been proven as an important circRNA molecule in nasopharyngeal carcinoma (NPC), however, its function still remains elusive. This study aims to discuss the biofunctions of hsa_circ_0066755 in NPC.

Methods: We detected the expression levels of hsa_circ_0066755 in NPC patients by quantitative real-time polymerase chain reaction ( $q R T-P C R)$, and the corresponding ROC curves were plotted. Functional experiments including CCK-8, colony formation, Transwell assay and Xenograft experiment were conducted. Bioinformatics analysis was performed to seek miRNAs which might have binding sites with hsa_circ_0066755. Luciferase reporter assays were finally carried out to verify the binding sites.

Results: We found significant increases of hsa_circ_0066755 in the plasma and tissues of the patients. Moreover, its levels were positively correlated with clinical staging $(P=0.019)$. The receiver operating characteristic (ROC) analysis showed that the area under the curves (AUCs) of tissue and plasma hsa_circ_0066755 for distinguishing NPC from non-cancerous controls were 0.8537 and 0.9044 , respectively. Both tissue and plasma hsa_circ_0066755 testing presented a comparable diagnostic accuracy to the magnetic resonance imaging (MRI). Our in-vitro experiment showed that the overexpression of hsa_circ_0066755 facilitated the growth, proliferation, clone formation, invasion and migration of CNE-1 NPC cells, while its down-regulation showed completely opposite effects. The xenograft experiment showed that exogenous hsa_circ_0066755 could significantly enhance the in-vivo tumorigenic ability of CNE-1 cells. Rescue assay further confirmed hsa_circ_0066755 as a tumor facilitator by sponging miR-65 1 .

Conclusions: Collectively, this study reported for the first time that hsa_circ_0066755 played a role of oncogene in NPC and could be used as an effective diagnostic marker for NPC, and that hsa_circ_0066755 / miR-65I axis also involved in the progression of NPC.
\end{abstract}

Key words: circular RNA, hsa_circ_0066755, nasopharyngeal carcinoma, CNE-1; miR-651, sponge

\section{Introduction}

NPC as a malignant tumor occurs in the nasopharyngeal mucosa, which is commonly reported in southern China and Southeast Asia [1]. Most of its pathological types are non-keratinizing, with a high degree of malignancy, aggressive invasion and metastasis profile [2]. Owing to the concealed location of the nasopharynx and complicated early symptoms, this disease may be easily missed or misdiagnosed at 
the early stage [3]. A majority of patients have reached the advanced stage by the time they consult a physician; thus, the prognosis is extremely poor [4]. Etiological studies have showed that the onset of NPC is associated with EBV infection, genetic susceptibility, dietary habits, and certain environmental and physicochemical factors, of which EBV plays an important role [5,6]. The progression, invasion, and metastasis of NPC are related to abnormal cell movement which is a dynamic process of interactions among various biological behaviors of tumor cells, such as adhesion, degradation, movement and angiogenesis [7, 8]. This complicated process is regulated by multiple factors, and involves multiple genes and signaling pathways $[9,10]$. Therefore, a clear interpretation of the occurrence and development of NPC from the perspective of genetic abnormality has great implications in identifying diagnostic and prognostic indicators, as well as new drug targets.

CircRNA as newly discovered endogenous non-coding RNA encompasses a closed loop structure without a 5'-end cap and 3'-end poly-A tail, primarily consists of exons and is abundant in eukaryotic cells $[11,12]$. CircRNA is highly conserved and stable, and contains richer transcripts than linear messenger RNA [13]. It regulates multiple life activities at the transcriptional and post-transcriptional levels. Studies have shown that circRNA acts as a microRNA (miRNA) sponge to inhibit miRNA activity and block the inhibition of its target by miRNA, thus regulating expressions of other related RNAs [14, 15]. In recent years, it has also been proven to be abnormally expressed in a variety of malignant tumors, affect the proliferation, differentiation, apoptosis and invasion of cancer cells, and participate in the onset and development of tumors [13, 16-18]. In our previous work, we conducted bioinformatics analysis to analyze the difference of circRNA expression profiles between NPC patients and non-cancer controls that from the GEO database, and found hsa_circ_0066755 was significantly up-regulated in the NPC tissues. Hsa_circ_0066755 locates at chr3:108295117-108298535, with a spliced length of 345 base. Hsa_circ_0066755 was generated from the coding exons of the KIAA1524 gene (data from circBase, http://circrna.org/). Currently, there is no previous evidence of detailed biofunctions and the corresponding mechanisms of hsa_circ_0066755 in NPC. The aim of this study was to investigate the expression of hsa_circ_0066755 in NPC patients and its potential clinical value. The biofunctions and molecular mechanisms in the disease were examined at the cellular and animal levels, aiming to identify new markers and therapeutic targets for future trials in patients.

\section{Materials and Methods}

\section{Patient specimens}

Carcinoma tissues from 30 NPC patients and biopsy tissues from 19 patients with nasal polyps were collected in The First Affiliated Hospital, and College of Clinical Medicine of Henan University of Science and Technology, so were the plasma samples (EDTA-K2 anticoagulant) from 86 NPC newly diagnosed patients and 86 healthy controls. All tissue samples of NPC patients were obtained by biopsy or surgery for the first time and confirmed by histopathological examination. The tissues and plasma samples were immediately frozen at liquid nitrogen or $-80^{\circ} \mathrm{C}$. The tumor stage classification was guided by the criteria of the 2017 edition for staging of nasopharyngeal carcinoma in China (The Chinese 2008 expert consensus on staging revision of nasopharyngeal carcinoma). This study was carried out with the approval from the Ethics Committee of The First Affiliated Hospital, and College of Clinical Medicine of Henan University of Science and Technology. Written informed consent was obtained from all participants. This study was conducted in accordance with the Declaration of Helsinki.

\section{Cell culture and transfection}

CNE-1 and CNE-2 cell lines were preserved in our laboratory and cultured in high glucose DMEM medium containing 10\% FBS (Hyclone). HEK-293a cell line was purchased from Chinese Academy of Sciences (Shanghai, China) and cultured in RPMI1640 culture solution containing 10\% FBS. Hsa_circ 0066755 sequence was obtained from the University of California Santa Cruz (UCSC) database (http:// genome.ucsc.edu/index.html), connected to the PLCDH-cir vector and packaged as a lentiviral recombinant vector. The Si-circ_0066755 shRNA was also packaged as a lentiviral vector. The target cells were transfected with miR-651 mimics or inhibitor via Lipofectamine $^{\mathrm{TM}} 2000$ (Thermo Fisher SCIENTIFIC) after completing synthesis.

\section{qRT-PCR}

Total RNA extracted from tissue and plasma was reversely transcribed into cDNA (Promega, USA) using RNAprep pure Tissue Kit (TIANGEN, China) and miRNeasy Serum/Plasma Purification Kit. RNA was isolated from the nucleus and cytoplasm using PARISTM Kit (Life Technologies, USA). The junction of hsa_circ_0066755 was cloned using the following divergent primers: Forward: '5- GCACTTTTCTTCAT GTCTTCACCA-3', reverse: '5- ACTATGGGCCAACA AGGTGAT-3'. The target gene was amplified by FastStart Universal SYBR Green Master (ROX) Kit 
(Roche, Switzerland). Each sample was analyzed 3 times. U6 (Forward: '5- CTACCAACACTGTAGAGG AGCC-3', reverse: '5-GCCTCGAAGCTCTCGGTCAT3 ') and GAPDH (Forward: '5- GGAGCGAGATCCCT CCAAAAT-3', reverse: '5-GGCTGTTGTCATACTTCT CATGG-3') were selected as reference genes for the $2^{-\Delta \Delta \mathrm{Ct}}$ relative quantification of $h s a_{-}$circ_0066755 in the nucleus and cytoplasm [19]. The median expression was used as a cutoff point for grouping the expression level of plasma hsa_circ_0066755 as "Low" and "High".

\section{Cell viability assay}

Cells in the logarithmic growth phase were harvested, and 2,000 cells were added into each well of a 96-well culture plate. The total volume was 100 $\mu \mathrm{L}$, and there were 2 parallel wells for each group. After incubation for 24, 48, 72 and 96 h, $10 \mu \mathrm{L}$ CCK-8 solution (DOJINDO) was added to each well before another $2 \mathrm{~h}$ incubation. The optical density (OD) of each well at $450 \mathrm{~nm}$ was measured by an ELIASA. The experiment was repeated three times to obtain the average.

\section{Colony formation assay}

Cells in the logarithmic growth phase were harvested and inoculated in a 6-well plate at a density of 800 cells/well. Then these cells were placed in a $37^{\circ} \mathrm{C}, 5 \% \mathrm{CO}_{2}$ atmosphere, and statically cultured for 2 to 3 weeks until macroscopic clonal cell clusters appeared in the culture dish. Acetic acid/methanol (1:3) of $5 \mathrm{~mL}$ was added for fixation for $15 \mathrm{~min}$. The cells were stained with Giemsa staining solution for $20 \mathrm{~min}$ and photographed under microscope. The number of cell colonies was counted, and a cluster containing $\geq 50$ cells was considered as a clone cluster.

\section{Wound healing assay}

Cells in the logarithmic growth phase were harvested and inoculated into a 6-well plate, and $5 \times 10^{5}$ cells were added into each well. The cells were placed in a $37{ }^{\circ} \mathrm{C}, 5 \% \mathrm{CO}_{2}$ atmosphere overnight and cultured until the fusion rate reached above $90 \%$. A sterile pipette tip $(200 \mu \mathrm{L})$ was used to perpendicularly scratch cells along a ruler. The migrated cells were washed with PBS and photographed at 0 and $48 \mathrm{~h}$ to calculate the number of migrated cells.

\section{Transwell assay}

Invasion experiment was performed by diluting Matrigel (Corning) at the ratio of 1:8 to cover the membrane (upper compartment) of Transwell chamber. Then the chamber was placed at $37^{\circ} \mathrm{C}$ for 30 min to allow Matrigel to polymerize into gel (Matrigel may be saved for the migration experiment). The cell density was adjusted to $5 \times 10^{5} / \mathrm{mL}$. Cell suspension (100 $\mu \mathrm{L} /$ well) were added to the upper compartment, and $500 \mu \mathrm{L} 20 \%$ FBS containing culture solution was added in the lower compartment of the 24-well plate for chemotaxis before culture in a humidified atmosphere of $5 \% \mathrm{CO}_{2}$ at $37^{\circ} \mathrm{C}$ for 24 to $48 \mathrm{~h}$. The cells and gel in Transwell were scraped off, fixed in $4 \%$ paraformaldehyde for $10 \mathrm{~min}$, stained with $0.1 \%$ crystal violet for $30 \mathrm{~min}$, and photographed under microscope to count the number of cells in at least 5 different fields of view.

\section{Immunoblotting}

In brief, total protein was extracted by RIPA lysis buffer (Beyotime), and was separated by $12 \%$ gel electrophoresis at constant pressure of $80 \mathrm{~V}$. The separated proteins were transferred to the PVDF membrane, and were blocked with TBST buffer containing $5 \%$ milk powder. The dilutions of the primary antibodies were: rabbit monoclonal Anti-Bcl-2 antibody (Abcam; 1:500), anti-caspase-3 (Beyotime; 1:500), and mouse monoclonal anti-human $\beta$-actin (Beyotime; 1:1000). Goat anti-rabbit IgG (1:10000) labeled with horseradish peroxidase (HRP) was utilized as the secondary antibody. ECL luminescent solution was added for the final imaging. Quantity ONE software was used for relative quantification of the proteins.

\section{Xenograft experiment}

The in-vivo study was carried strictly following the protocols of Guide for the Care and Use of Laboratory Animals of the NIH (Bethesda, MD). Sixweek-old BALB/C nude mice with a weight of 18-20 g were selected. The cell concentration of each group was adjusted to $2 \times 10^{7}$ cells $/ \mathrm{mL}$, and $100 \mu \mathrm{L}$ cells were subcutaneously inoculated into the middle portion of the inguinal region of each mouse. Subcutaneous tumor was observed around 1 week after inoculation. According to the tumorigenic ability of CNE-1 cells, tumor weights were measured at the end of the fourth week of the experiment. This study was approved by the Ethics of The First Affiliated Hospital, and College of Clinical Medicine of Henan University of Science and Technology.

\section{Dual luciferase reporter assay}

MiRNA molecules and binding sites interacting with hsa_circ_0066755 were predicted by Circular RNA Interactome (https://circinteractome.nia.nih. gov/). The wild-type (wt) and mutant (mut) hsa_circ_ 0066755 sequences were separately designed and cloned into the pmirGLO vector (Promega). Its recombinant vector and miR-651 mimics were cotransfected to HEK-293a cells via Lipofectamine ${ }^{\mathrm{TM}}$ 2000 (Thermo Fisher SCIENTIFIC) according to its 
protocol. The cells were lysed after $24 \mathrm{~h}$ of culture and luciferase activity was measured.

\section{Statistical analyses}

Statistical analysis was performed using SPSS 16.0 software. Measurement data were expressed as mean \pm standard deviation (SD). If the assumptions of normality and homoscedasticity were satisfied, Student's $t$-test was conducted to compare the differences between the two groups. Correlations between hsa_circ_0066755 expression and clinical pathological characterstics were analyzed by chi-square test or Spearman correlation analysis. The diagnostic value was evaluated by drawing a ROC curve. A $P$ value of $<0.05$ was considered for statistical significance.

\section{Results}

\section{Hsa_circ_0066755 was up-regulated in NPC patients}

Plasma levels of $h s a \_c i r c \_0066755$ in 86 newly diagnosed NPC patients and 86 healthy controls were tested by qRT-PCR. As a result, the NPC group presented a significantly higher level of hsa_circ_ 0066755 than the control group (Figure 1A and 1B). Hsa_circ_0066755 levels in 16 pairs of NPC tissues were determined, which also revealed a significant up-regulation in the tissues (Figure 1C and 1D). Furthermore, stage I and II NPC patients showed lower plasma and tissue levels of hsa_circ_0066755 than those in stage III and IV (Figure 1E and 1F). Correspondingly, correlation analysis confirmed that plasma hsa_circ_0066755 expression was positively correlated with clinical staging $(P=0.019)$ (Table 1). ROC analysis showed that the AUCs of plasma and tissue hsa_circ_0066755 for distinguishing noncancerous controls from NPC subjects were 0.8537 and 0.9044 , respectively (Figure $1 \mathrm{G}$ and $1 \mathrm{H}$ ). We followed up 24 of 86 patients (including all 16 tissue donors) for more than 3 months. The results showed that 23 patients with positive MRI findings were diagnosed as NPC by histopathological examination. The diagnostic accuracy of MRI and plasma and tissue hsa_circ_0066755 was $95.83 \%(23 / 24), 83.33 \%(20 / 24)$ and $87.50 \%(14 / 16)$ (Table 2), suggesting that plasma and tissue hsa_circ_0066755 were very useful in the diagnosis of NPC.

\section{Overexpression of hsa_circ_0066755 promoted the proliferation and invasion of CNE-1 cells}

Results of qRT-PCR showed that CNE-1 and CNE-2 cells presenting certain levels of hsa_circ 0066755, which were significantly higher than those in
HEK-293a cells (Figure 2A). CNE-1 cells that moderately expressed $h s a \_c i r c \_0066755$ were selected as the target cells. After infected with PLCDH-circ 0066755, hsa_circ_0066755 levels in CNE-1 cells increased by 7.78 folds (Figure 2B). The CCK- 8 assay showed that up-regulated exogenous hsa_circ_0066755 expression could significantly promote the growth of CNE-1 cells (Figure 2C and $2 \mathrm{D})$. The clone formation experiment showed that the number of cloned cells in the PLCDH-circ_0066755 group was significantly higher than that in the PLCDH-vector group $(P<0.01)$ (Figure 2E). Scratch wound healing and Transwell chamber experiments showed that the number of invasive and migrated cells in the PLCDH-circ_0066755 group was significantly higher than that in the PLCDH-vector group (Figure $2 \mathrm{~F}$ and $2 \mathrm{G}$ ). These results suggested that the overexpression of $h s a \_c i r c \_0066755$ promoted the growth, clone formation, invasion and migration of CNE-2 cells. Thus, we further designed an in vivo experiment. The tumor formation experiment of nude mice showed that the PLCDH-circ_0066755 group presented significantly heavier weights of tumors and evidently stronger tumorigenic abilities than the PLCDH-vector group (Figure 2H).

Table 1. Clinical characteristics of plasmic hsa_circ_0066755 expression in NPC

\begin{tabular}{llllll} 
Clinicopathological features & $\begin{array}{l}\text { Total } \\
(\mathrm{n}=86)\end{array}$ & Low $(\mathrm{n}=33)$ & $\begin{array}{l}\text { High } \\
(\mathrm{n}=53)\end{array}$ & $\chi^{2}$ value & $P$ value \\
\hline Gender & 66 & 25 & 41 & 0.029 & 0.864 \\
Male & 20 & 8 & 12 & & \\
$\begin{array}{l}\text { Female } \\
\text { Age (years) }\end{array}$ & & & & & \\
$\leq 45$ & 37 & 12 & 25 & & \\
$>45$ & 49 & 21 & 28 & & \\
Clinical stage & & & & 5.469 & 0.019 \\
I-II & 41 & 21 & 20 & & \\
III-IV & 45 & 12 & 33 & & \\
Pathological type & & & & 1.625 & 0.202 \\
Nonkeratinizing & 85 & 32 & 53 & & \\
Keratinizing & 1 & 1 & 0 & & \\
Lymphatic metastasis & & & & 1.903 & 0.168 \\
Yes & 24 & 12 & 12 & & \\
No & 62 & 21 & 41 & & \\
\hline
\end{tabular}

Table 2. Diagnostic accuracy of plasma and tissue hsa_circ_0066755 and MRI in confirming NPC

\begin{tabular}{lllll}
\hline Groups & Total size & $\begin{array}{l}\text { Missed } \\
\text { diagnosis }(\%)\end{array}$ & $\begin{array}{l}\text { Misdiagnosis } \\
(\%)\end{array}$ & $\begin{array}{l}\text { Diagnostic } \\
\text { accuracy }(\%)\end{array}$ \\
\hline $\begin{array}{l}\text { Plasmic } \\
\text { hsa_circ_0066755 }\end{array}$ & 24 & $4(16.67)$ & 0 & $20(83.33)$ \\
$\begin{array}{l}\text { Tissue } \\
\text { hsa_circ_0066755 } \\
\text { MRI }\end{array}$ & 16 & $2(12.5)$ & 0 & $14(87.5)$ \\
\hline
\end{tabular}



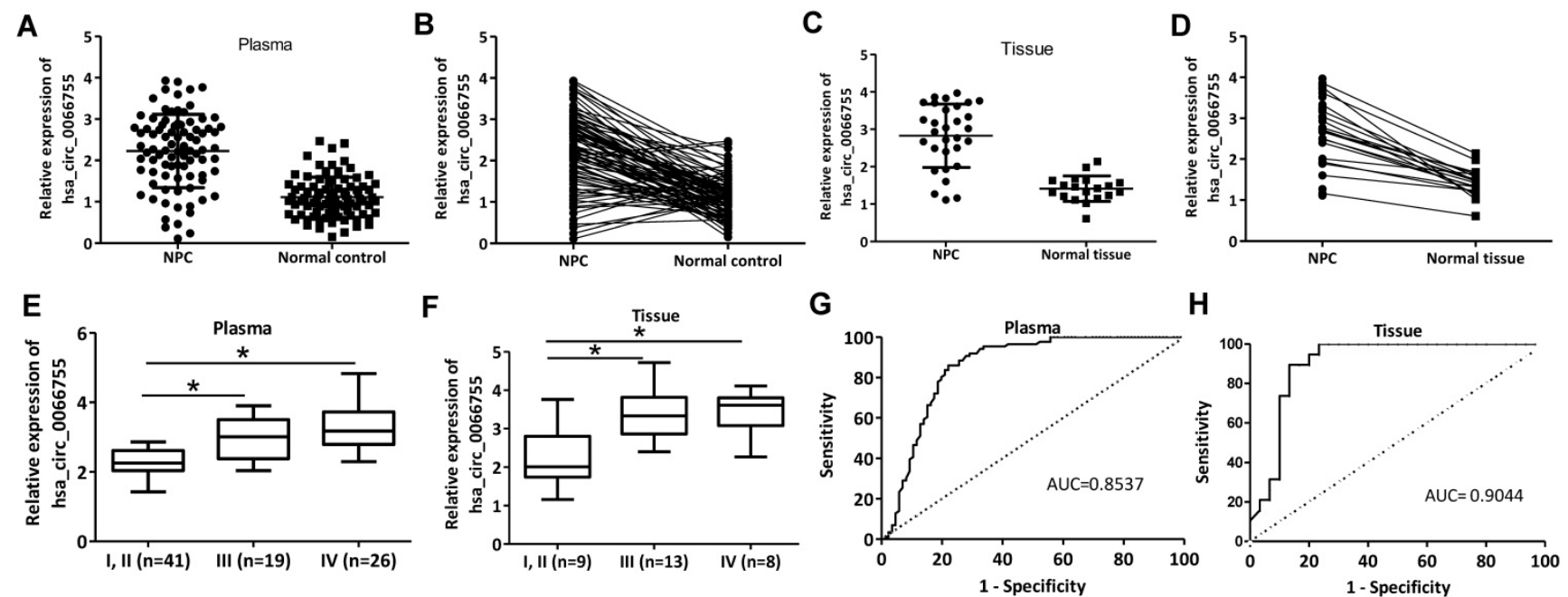

Figure 1. Plasma and tissue levels of hsa_circ_0066755 in NPC patients and the clinical significance. (A,B) Plasma and (C, D) tissue levels of hsa_circ_0066755 in NPC patients detected by qRT-PCR. (E, F) Plasmic or tissue levels of hsa_circ_0066755 were higher in stage III and IV NPC patients than those in stage I and II patients. The plotted ROC curves of $(\mathrm{G})$ plasma and $(\mathrm{H})$ tissue hsa_circ_0066755 in the diagnosis of NPC. $* P<0.05$.

A

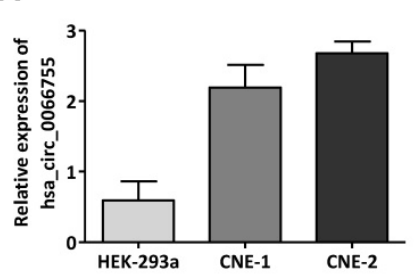

E
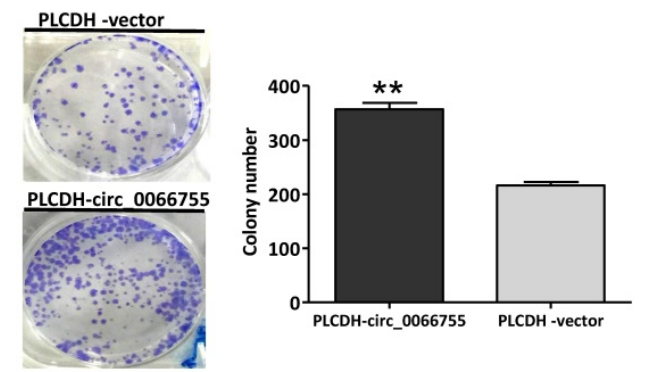

G

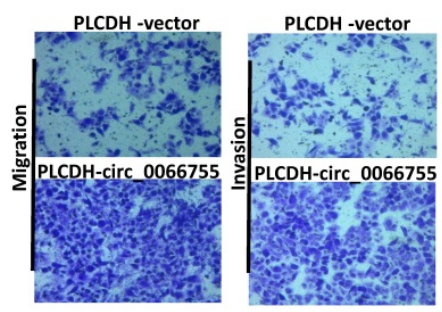

B

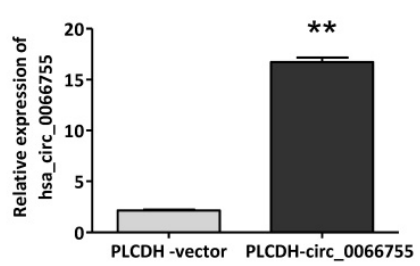

C

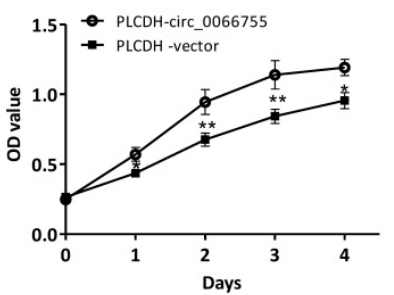

D

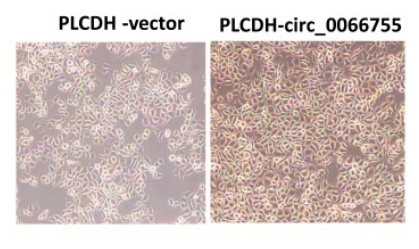

$\mathbf{F}$
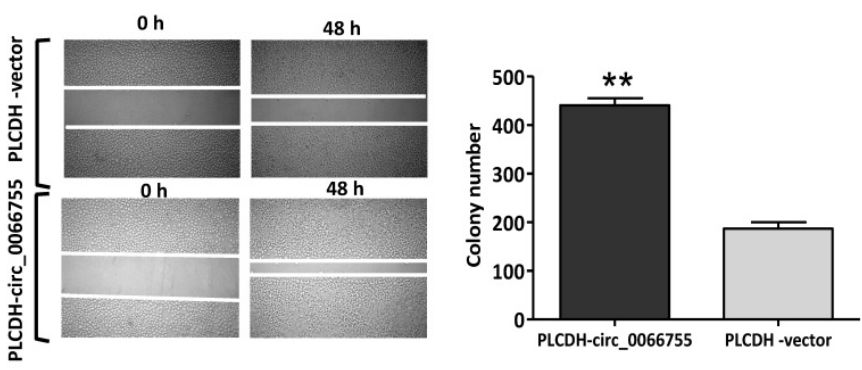

H
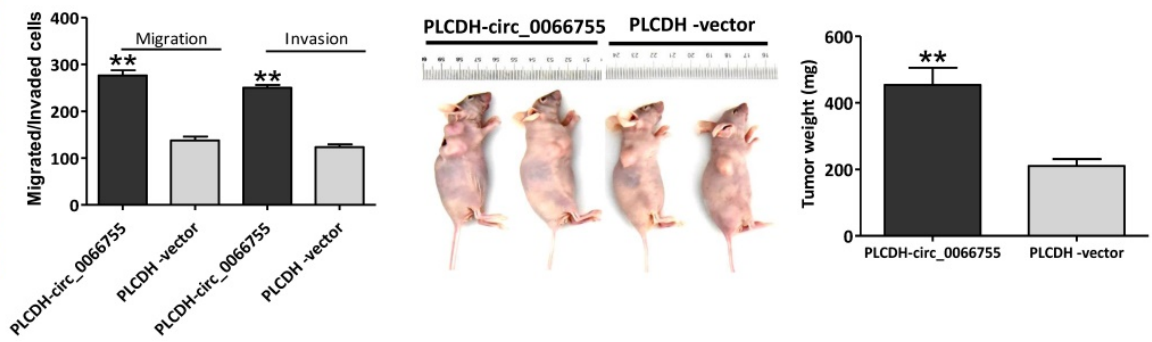

Figure 2. Up-regulation of hsa_circ_0066755 promoted the growth, proliferation, invasion and migration of CNE-1 cells. (A) Basal levels of $h s a \_c i r c \_0066755$ in HEK-293a, CNE-1 and CNE-2 cell lines. (B) The expression of total hsa_circ_0066755 in CNE-1 cells following infection. (C, D) Cell viability assessed by CCK-8 assay, and cells morphology and growth condition was imaged at $72 \mathrm{~h}$ following infection $(\times 200)$. (E) Colony formation assay. $(\mathrm{F})$ Wound healing migration assay. Migrated cells were counted at $48 \mathrm{~h}$ after transfection. (G) Transwell invasion assay. Migrated or invaded cells were counted at $48 \mathrm{~h}$ following transfection with or without Matrigel. (H) Xenograft experiment. Tumors were weighed at the endpoint of the experiment. $* P<0.05$, $* * P<0.01$ vs. PLCDH-vector group.

\section{Hsa_circ_0066755 silencing inhibited the proliferation and invasion of CNE-1 cells}

Results of qRT-PCR indicated that after silencing levels of hsa_circ_0066755 in CNE-1 cells were significantly down-regulated (Figure 3A). The CCK-8 test showed that down-regulating endogenous $h s a_{-}$ circ_0066755 expression could obviously inhibit the growth of CNE-1 cells (Figure 3B and 3C). As illustrated in Figure 3D, the number of cloned cells in 
the Si-circ_0066755 group was evidently lower than that in the Si-vector group $(P<0.05)$. Scratch-wound healing and Transwell chamber experiments revealed that the number of invasive and migrated cells in the Si-circ_0066755 group was much lower than that in the Si-vector group (Figure $3 \mathrm{E}$ and $3 \mathrm{~F}$ ). Moreover, immunoblotting showed that expression of Bcl-2 protein in Si-circ_0066755 group were downregulated, along with an activation of procaspase-3, suggesting that $h s a \_c i r c \_0066755$ silencing may induce apoptosis in CEN1 cells (Figure 3G). Collectively, these results suggested that down-regulating endogenous hsa_circ_0066755 expression could inhibit the growth, clone formation, migration and invasion of CNE-1 cells.

\section{Hsa_circ_0066755 regulated NPC progression by sponging miR-65 1}

As hsa_circ_0066755 has been proven to be located in the cytoplasm of CNE-1 cells, we assumed that hsa_circ_0066755 possibly acts as the sponge of miRNA (Figure 4A). Based on the predicted results in our bioinformatic analysis, 17 kinds of miRNAs potentially interacted with hsa_circ_0066755 (Table 3). Four were identified to have significant inhibitory effects on luciferase activity using the luciferase reporter gene screening system for miRNA target genes, of which miR-651 showed superior inhibitory effects over others. To verify the effects of $h s a_{-}$ circ_0066755 on miR-651, we silenced the gene by transfecting si-hsa_circ_0066755 shRNA vectors into CNE-1 cells. As a result, miR-651 expressions obviously increased (Figure 4B). Besides, the dual luciferase reporter assay revealed that luciferase activity in CNE-1 and HEK-293a cells co-transfected with miR-651 mimics in the hsa_circ_006675-wt group was significantly lowered compared with the negative control group, while the hsa_circ_006675-mut group presented nonsignificant changes in luciferase activity (Figure $4 \mathrm{C}$ and $4 \mathrm{D}$ ). Using the miR-651 mimic and inhibitor, we successfully proved the positive and negative effects of the interaction between hsa_circ 0066755 and miR-651 on the biological characteristics of CNE- 1 cells. The CCK-8 test alongside the clone formation test, the scratch assay and the Transwell experiment showed that the circ_0066755+mimic group presented weaker growth of CNE-1 cells, a lower number of cloned cells and weaker cell invasion and migration than the circ_0066755 group; however, the Si-circ_0066755+inhibitor group revealed evidently stronger growth of CNE-1 cells, a higher number of cloned cells and stronger cell invasion and migration than the Si-circ_0066755 group (Figure 4E to $4 \mathrm{~L})$.
Table 3. The predicted miRNAs that may target with hsa_circ_0066755

\begin{tabular}{|c|c|c|}
\hline Predicted miRNAs & Binding site with $h s a \_c i r c \_0066755$ & $\begin{array}{l}\text { Context+ score } \\
\text { percentile }\end{array}$ \\
\hline hsa-miR-1224-3p & $5^{\prime} \ldots$ UCCACCC ...3' & 96 \\
\hline hsa-miR-1243 & $5^{\prime} \ldots$ UAGGUCA....3' & 98 \\
\hline hsa-miR-1261 & $5^{\prime} \ldots$ AAUAGGU $\ldots 3^{\prime}$ & 97 \\
\hline$h s a-m i R-140-3 p$ & $5^{\prime} \ldots$ GACACCA $\ldots 3^{\prime}$ & 98 \\
\hline hsa-miR-323-3p & $5^{\prime} \ldots$ CAUUACA....3' & NA \\
\hline$h s a-m i R-361-3 p$ & $5^{\prime} \ldots$ ACСССС $\ldots 3^{\prime}$ & 92 \\
\hline hsa-miR-488 & $5^{\prime} \ldots$ GAAAGU $\ldots 3^{\prime}$ & 87 \\
\hline hsa-miR-545 & $5^{\prime} \ldots$ AACGAC $\ldots 3^{\prime}$ & 93 \\
\hline hsa-miR-579 & $5^{\prime} \ldots$ UUUACU $\ldots 3^{\prime}$ & 90 \\
\hline hsa-miR-580 & $5^{\prime} \ldots$ UAAGAGU...3' & 99 \\
\hline hsa-miR-587 & $5^{\prime} \ldots$ UACCUU ....3' & 87 \\
\hline hsa-miR-606 & $5^{\prime} \ldots$ UCAUCAA.... $3^{\prime}$ & 97 \\
\hline hsa-miR-624 & $5^{\prime} \ldots$ UGGAACA....3' & 94 \\
\hline hsa-miR-651 & $5^{\prime} \ldots$ UAGGAUU....3' & 99 \\
\hline$h s a-m i R-885-5 p$ & $5^{\prime} \ldots$ AUUACC....3' & 89 \\
\hline$h s a-m i R-890$ & $5^{\prime} \ldots$..GGUUCA ....3' & 92 \\
\hline hsa-miR-936 & $5^{\prime} \ldots$ GAUGAC $\ldots 3^{\prime}$ & 89 \\
\hline
\end{tabular}

\section{Discussion}

Though the onset is regional, NPC owns a high incidence in an Asian population [1, 4]. Because of the non-specific symptoms at the early stage, most initial confirmed cases have entered the late stage with a high likelihood of distal metastasis and a high risk local recurrence $[2,3,5]$. Better understanding of mechanisms behind its onset and development and identification of reliable markers for NPC are essential to the clinical diagnosis and treatments. CircRNA as a special class of non-coding RNA molecules has been a latest hotspot [11, 12]. It may act as a constituent of competitive RNA (ceRNA) to inhibit the activity of miRNA, regulating gene transcription, translation and other functions thereby [20-22]. This study has explored the biofunctions of hsa_circ_0066755 in NPC and the corresponding mechanisms, and discussed its potential clinical value as a diagnostic biomarker for NPC.

At present, very few studies have focused on circRNAs in NPC. Some have reported that circMAN1A2 expression is elevated in NPC tissue and is highly effective in its diagnosis $(\mathrm{AUC}=0.91)$ [23]. Shuai et al. have found that highly expressed circRNA_0000285 in NPC is associated with tumor size, tumor cell differentiation, lymph node metastasis, distant metastasis, etc., which may be used as an independent prognostic indicator [24]. This study shows that plasma and tissue hsa_circ_0066755 expressions evidently increase in NPC patients and are positively correlated with tumor progression and TNM staging, which in turn are highly effective in the diagnosis of NPC with AUCs of 0.8537 and 0.9044, respectively. Moreover, plasma hsa_circ_0066755 is highly consistent with MRI in terms of the diagnosis of NPC. An easier access to blood samples with a less- 
invasive sampling process has made plasma $h s a_{-}$ circ_0066755 as one of the promising diagnostic indicators of NPC.

Therefore, to the best of our knowledge, we have for the first time explored the biofunctions of $h s a_{-}$ circ_0066755 in NPC, pro and con. It is found that exogenous $h s a \_c i r c \_0066755$ can significantly improve the growth, clone formation, and invasion and migration of CNE-1 cells, while its down-regulation leads to the opposite results. Our in-vivo experiment further indicates that $h s a \_c i r c \_0066755$ can improve the tumorigenic ability of CNE-1 cells in nude mice. To sum up, this study proves that hsa_circ_0066755 functions as an oncogene in NPC, which correlates with its clinical progression. A study by Zhu et al. suggests that down-regulating circ-ZNF609 expression in NPC 5-8F and HONE-1 cells can significantly repress cell proliferation, invasion and migration [25], which is similar to our findings.

Studies have shown that circRNA can regulate expressions of its target genes via sponging miRNA, which has been proven in multiple malignant tumors and their progressions [26-29]. The qRT-PCR analysis in our study has confirmed that hsa_circ_0066755 is mainly distributed in the cytoplasm of CNE-1 and HEK-293a cells. Thus, we have assumed that hsa_circ_ 0066755 may play the role as a miRNA sponge. Based on the bioinformatic analysis, we have predicted that there are 46 potential miRNA molecules interacting with hsa_circ_0066755. Through the screening of the luciferase reporter gene screening system for miRNA target genes, 4 present significantly inhibitory effects on luciferase activity, and miR-651 is the most prominent factor. To decrease the confounding factors, we selected human HEK293a and CNE-1 cells for the study of hsa_circ_0066755 and miR-651 interaction. Our study further verified that hsa_circ 0066755 interacts with $m i R-651$ and they can affect the biological characteristics of CNE-1 cells.

Nevertheless, this study still has limitations, including a small sample size, only one cell line used for verifying cell functions, a lack of deep investigation of the possible apoptosis effects, and failure to confirm the hsa_circ_0066755 silencing experiments in-vivo, etc. Our research team will constantly improve the experiments above to overcome these limitations in the future.
A

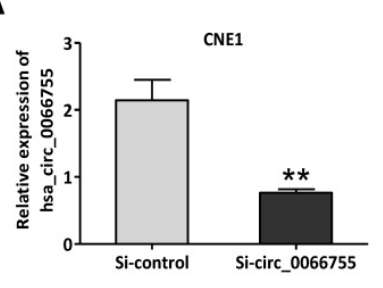

B

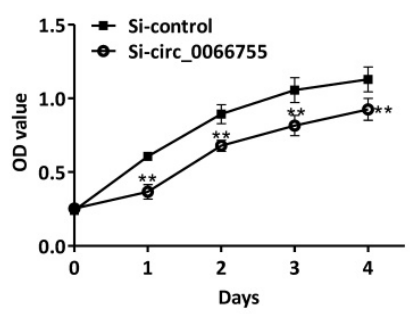

C

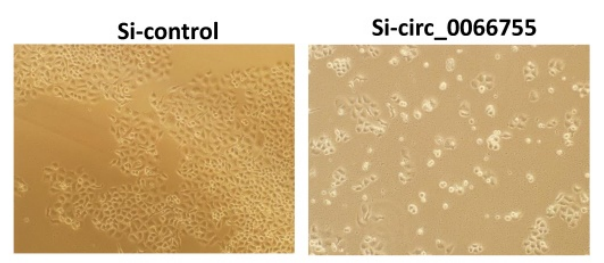

D

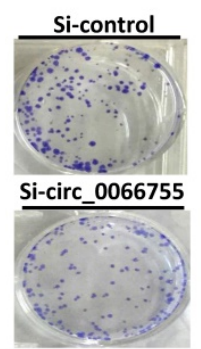

$\mathbf{F}$

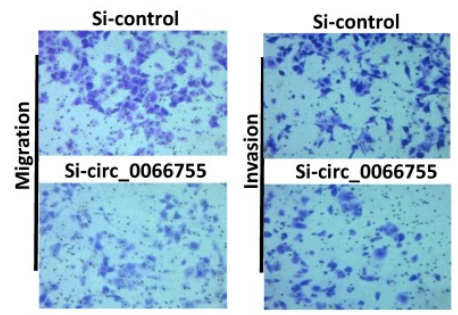

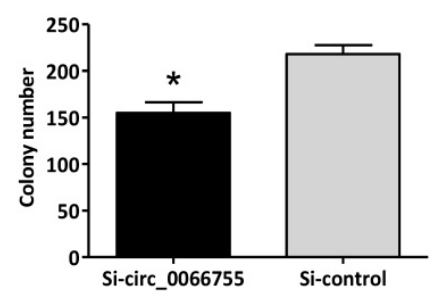

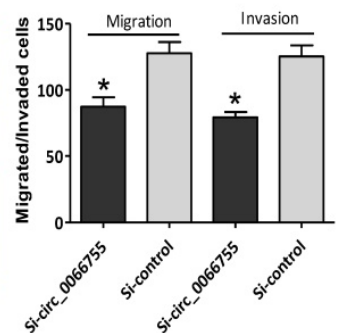

$\mathbf{E}$

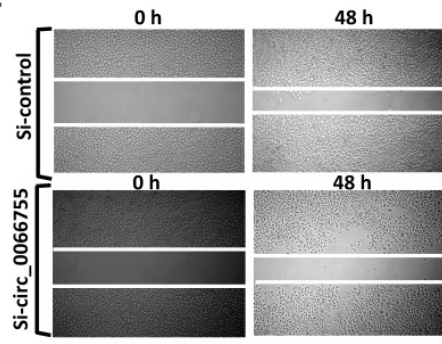

G

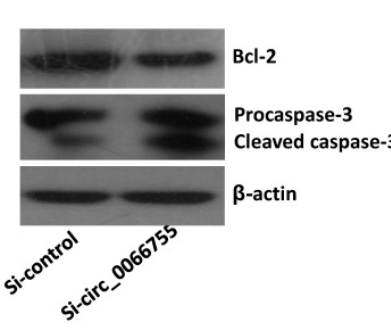

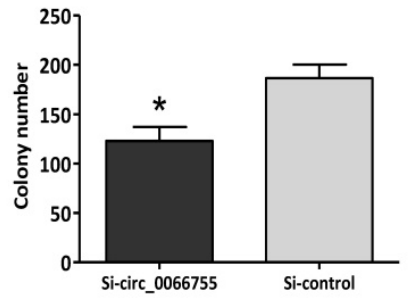

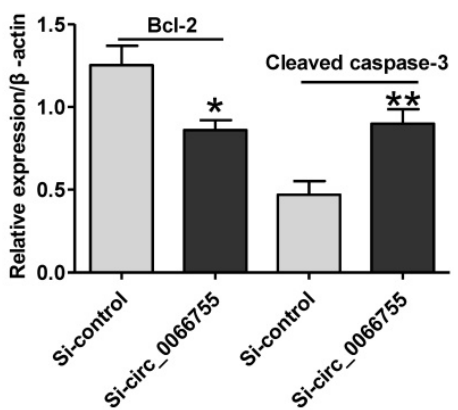

Figure 3. Hsa_circ_0066755 silencing inhibited the proliferation and invasion of CNE-1 cells. (A) The expression of hsa_circ_0066755 in CNE-1 cells following transfection with the si-hsa circ 0066755 vector. (B) Cell viability assessed by CCK-8 assay. (C) Morphological changes of CNE-1 cells after infected with the Si- circ 0066755 shRNA vector at 72h. (D) Colony formation assay. (E) Wound healing migration assay. Migrated cells were counted at $48 \mathrm{~h}$ after silencing. (F) Transwell invasion assay. Migrated or invaded cells were counted at $48 \mathrm{~h}$ after transfection with or without Matrigel. $(\mathrm{G})$ Expression of apoptosis related proteins detected by immunoblotting. $* P<0.05, * * P<0.01$ vs. Si-control group. 
A

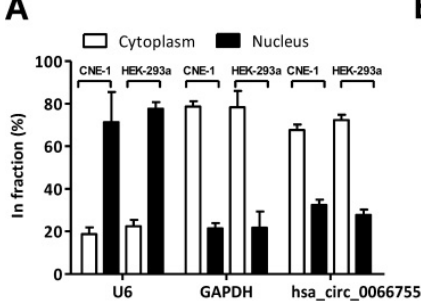

E

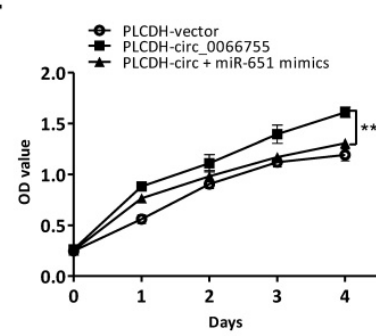

I

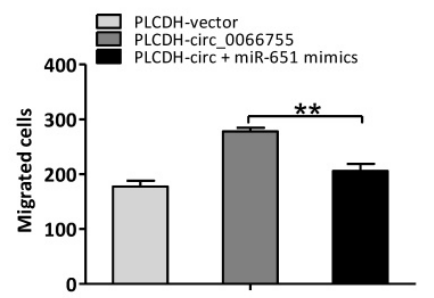

B

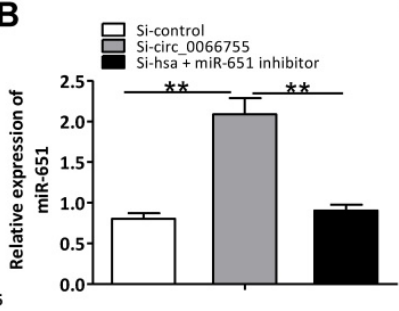

F

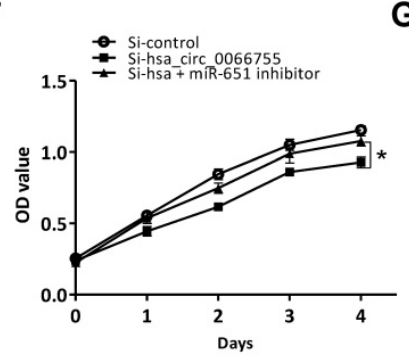

J

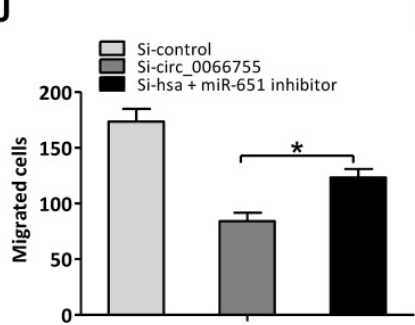

C

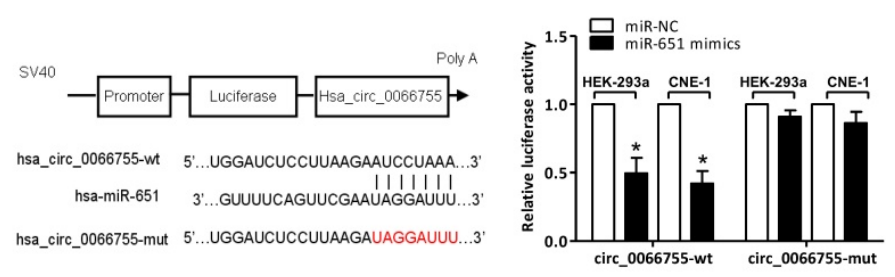

G

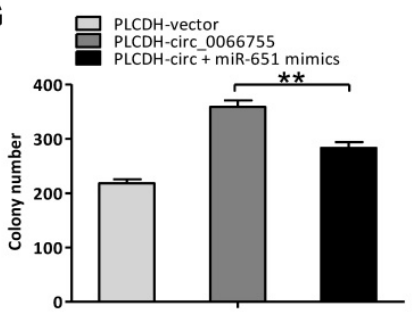

K

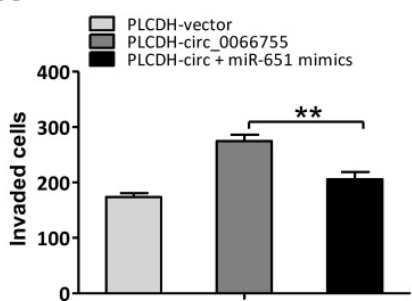

H

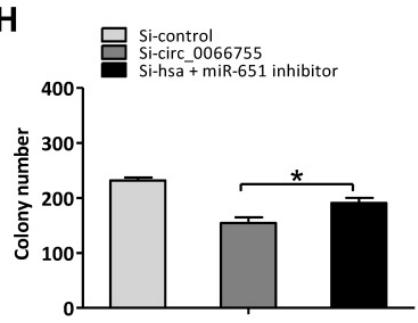

$\mathbf{L}$

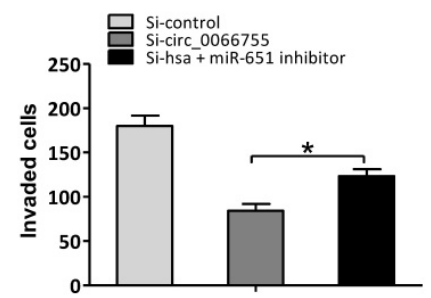

Figure 4. Hsa_circ_0066755 acted as the miR-65I sponge to regulate NPC progression. (A) Distribution of hsa_circ_0066755 in nuclear and cytoplasmic fractions of CNE-1 cells. (B) Hsa_circ_0066755 silencing could increase miR-65I expression. (C, D) Luciferase assay of HEK-293a and CNE-1 cells co-transfected with the miR-65I mimic and luciferase reporter containing hsa_circ_0066755-wild-type sequences (cirRNA-wt) or -mutant sequences (cirRNA-mut). (E, F) Cell viability of CNE-1 cells assessed by CCK-8 assay. $(\mathrm{G}, \mathrm{H})$ Colony formation assay. $(\mathrm{I}, \mathrm{J})$ Wound healing migration assay. $(\mathrm{K}, \mathrm{L})$ Transwell invasion assay. $* P<0.05, * * P<0.01$.

In conclusion, hsa_circ_0066755 expression is up-regulated in NPC and is associated with the progression of NPC. Plasma and tissue hsa_circ_ 0066755 is very effective in the diagnosis of NPC, of which plasma hsa_circ_0066755 can be promoted as a diagnostic biomarker due to an easier access to blood samples than tumor tissues. Besides, hsa_circ_0066755 involves as the miR-651 sponge in the growth, proliferation, invasion and migration of NPC cells.

\section{Abbreviations}

AUC: area under the curve; ceRNA: competitive RNA; circRNAs: circular RNAs; miRNA: microRNA; MRI: magnetic resonance imaging; NPC: nasopharyngeal carcinoma; qRT-PCR: quantitative real-time polymerase chain reaction; ROC: receiver operating characteristic.

\section{Acknowledgements}

The authors would like to thank Dr. Panke Su for the constructive suggestions to this article.

\section{Competing Interests}

The authors have declared that no competing interest exists.

\section{References}

1. Chen YP, Chan ATC, Le QT, Blanchard P, Sun Y, Ma J. Nasopharyngeal carcinoma. Lancet (London, England). 2019; 394: 64-80.

2. Lee HM, Okuda KS, Gonzalez FE, Patel V. Current Perspectives on Nasopharyngeal Carcinoma. Advances in experimental medicine and biology. 2019; 1164: 11-34.

3. Petersson F. Nasopharyngeal carcinoma: a review. Seminars in diagnostic pathology. 2015; 32: 54-73.

4. Kamran SC, Riaz N, Lee N. Nasopharyngeal carcinoma. Surgical oncology clinics of North America. 2015; 24: 547-61.

5. Tsao SW, Tsang CM, Lo KW. Epstein-Barr virus infection and nasopharyngeal carcinoma. Philosophical transactions of the Royal Society of London Series B, Biological sciences. 2017; 372 .

6. Zhang J, Jia L, Tsang CM, Tsao SW. EBV Infection and Glucose Metabolism in Nasopharyngeal Carcinoma. Advances in experimental medicine and biology. 2017; 1018: 75-90.

7. Yoshizaki T. Promotion of metastasis in nasopharyngeal carcinoma by Epstein-Barr virus latent membrane protein-1. Histology and histopathology. 2002; 17: 845-50.

8. Yoshizaki T, Ito M, Murono S, Wakisaka N, Kondo S, Endo K. Current understanding and management of nasopharyngeal carcinoma. Auris, nasus, larynx. 2012; 39: 137-44.

9. Ooft ML, Braunius WW, Heus P, Stegeman I, van Diest PJ, Grolman W, et al. Prognostic significance of the EGFR pathway in nasopharyngeal carcinoma: a systematic review and meta-analysis. Biomarkers in medicine. 2015; 9: 997-1010.

10. Zhao CX, Zhu W, Ba ZQ, Xu HJ, Liu WD, Zhu B, et al. The regulatory network of nasopharyngeal carcinoma metastasis with a focus on EBV, lncRNAs and miRNAs. American journal of cancer research. 2018; 8: 2185-209.

11. Chen LL, Yang L. Regulation of circRNA biogenesis. RNA biology. 2015; 12: 381-8.

12. Li X, Yang L, Chen LL. The Biogenesis, Functions, and Challenges of Circular RNAs. Molecular cell. 2018; 71: 428-42.

13. Patop IL, Wust S, Kadener S. Past, present, and future of circRNAs. The EMBO journal. 2019; 38: e100836. 
14. Kulcheski FR, Christoff AP, Margis R. Circular RNAs are miRNA sponges and can be used as a new class of biomarker. Journal of biotechnology. 2016; 238: 42-51.

15. Panda AC. Circular RNAs Act as miRNA Sponges. Advances in experimental medicine and biology. 2018; 1087: 67-79.

16. Kristensen LS, Hansen TB, Veno MT, Kjems J. Circular RNAs in cancer: opportunities and challenges in the field. Oncogene. 2018; 37: 555-65.

17. Patop IL, Kadener S. circRNAs in Cancer. Current opinion in genetics \& development. 2018; 48: 121-7.

18. Zhao X, Cai Y, Xu J. Circular RNAs: Biogenesis, Mechanism, and Function in Human Cancers. International journal of molecular sciences. 2019; 20.

19. Bao X, Wu H, Zhu X, Guo X, Hutchins AP, Luo Z, Song H, Chen $Y$, Lai K, Yin M, Xu L, Zhou L, Chen J, Wang D, Qin B, Frampton J, Tse HF, Pei D, Wang H, Zhang B, Esteban MA. The p53-induced lincRNA-p21 derails somatic cell reprogramming by sustaining $\mathrm{H} 3 \mathrm{~K} 9 \mathrm{me} 3$ and $\mathrm{CpG}$ methylation at pluripotency gene promoters. Cell Res. 2015 Jan;25(1):80-92.

20. Qi X, Zhang DH, Wu N, Xiao JH, Wang X, Ma W. ceRNA in cancer: possible functions and clinical implications. Journal of medical genetics. 2015; 52: 710-8.

21. Zhong Y, Du Y, Yang X, Mo Y, Fan C, Xiong F, et al. Circular RNAs function as ceRNAs to regulate and control human cancer progression. Molecular cancer. 2018; 17: 79 .

22. Qu S, Liu Z, Yang X, Zhou J, Yu H, Zhang R, et al. The emerging functions and roles of circular RNAs in cancer. Cancer letters. 2018; 414: 301-9.

23. Fan CM, Wang JP, Tang YY, Zhao J, He SY, Xiong F, et al. circMAN1A2 could serve as a novel serum biomarker for malignant tumors. Cancer science. 2019; 110: $2180-8$.

24. Shuai M, Hong J, Huang D, Zhang X, Tian Y. Upregulation of circRNA_0000285 serves as a prognostic biomarker for nasopharyngeal carcinoma and is involved in radiosensitivity. Oncology letters. 2018; 16: 6495-501.

25. Zhu L, Liu Y, Yang Y, Mao XM, Yin ZD. CircRNA ZNF609 promotes growth and metastasis of nasopharyngeal carcinoma by competing with microRNA-150-5p. European review for medical and pharmacological sciences. 2019; 23: 2817-26.

26. He J, Xie Q, Xu H, Li J, Li Y. Circular RNAs and cancer. Cancer letters. 2017; 396: 138-44.

27. Ng WL, Mohd Mohidin TB, Shukla K. Functional role of circular RNAs in cancer development and progression. RNA biology. 2018; 15: 995-1005.

28. Militello G, Weirick T, John D, Doring C, Dimmeler S, Uchida S. Screening and validation of lncRNAs and circRNAs as miRNA sponges. Briefings in bioinformatics. 2017; 18: 780-8.

29. Lin $X$, Chen Y. Identification of Potentially Functional CircRNA-miRNA-mRNA Regulatory Network in Hepatocellular Carcinoma by Integrated Microarray Analysis. Medical science monitor basic research. 2018; 24: 70-8. 